\title{
Méthodologie d'identification du comportement mécanique des mousses hyperélastiques par mesures de champs et méthode inverse
}

\author{
Fabrice Pierron ${ }^{\mathrm{a}}$, Baoqiao Guo et René Rotinat \\ Laboratoire de Mécanique et Procédés de Fabrication, Arts et Métiers ParisTech (ENSAM), Rue St Dominique, BP 508, \\ 51006 Châlons-en-Champagne, France
}

Reçu le 28 août 2008, accepté le 9 avril 2009

\begin{abstract}
Résumé - Cet article présente une méthodologie d'identification du comportement de mousses polymères hyperélastiques à partir de mesures de champs par corrélation d'images et de la technique d'identification dite méthode des champs virtuels. Dans un premier temps, les constantes élastiques à faible déformation sont identifiées à partir d'un essai de flexion/cisaillement. Ensuite, une loi hyperélastique de type Ogden est identifiée à partir d'un essai de compression uniaxiale. On observe une forte localisation des déformations en raison de l'effondrement élastique des cellules de la mousse, confirmant qu'une approche basée sur la réponse globale force/déplacement ne permettra pas l'identification d'un comportement matériau (effet de structure). Cette étude préliminaire montre le potentiel de la démarche même s'il reste encore beaucoup à faire pour fiabiliser cette approche.
\end{abstract}

Mots clés : Mousse polymère / hyperélasticité / mesure de champs / identification inverse / corrélation d'images / méthode des champs virtuels

\begin{abstract}
Identification methodology of the mechanical behaviour of hyperelastic foams using full-field measurements and an inverse method. This paper presents a methodology aimed at the identification of the mechanical behaviour of hyperelastic low density polymeric foams using digital image correlation and the Virtual Fields Method. First, a simple bending/shear test enables the identification of the elastic parameters at low strain levels. Then, a uniaxial compression test is performed to identify the parameters of an Ogden-type law (hyperelastic). The strain fields exhibit strong localization caused by the elastic collapse of the cells clearly indicating that an approach based on global force/displacement curves will fail to provide intrinsic material parameters (structural effect). This is still very much a preliminary study but it clearly shows the potential of the approach for this type of materials.
\end{abstract}

Key words: Polymeric foam / hyperelasticity / full-field measurements / inverse identification / digital image correlation / virtual fields method

\section{Introduction}

Les mousses polymères basse densité sont largement utilisées dans des applications nécessitant, entre autres, une bonne absorption d'énergie d'impact. Leur caractérisation mécanique est néanmoins rendue difficile par leur comportement hyperélastique et les phénomènes de localisation très aigus liés à l'écrasement des cellules en compression. Les méthodologies d'identification classiques à base d'essais homogènes ou simples ne sont donc pas adaptées.

${ }^{a}$ Auteur pour correspondance :

fabrice.pierron@chalons.ensam.fr
La littérature présente encore très peu de tentatives d'utilisation de mesures de champs cinématiques pour traiter ce problème. Quelques références récentes sont disponibles mais les mesures servent principalement à des observations qualitatives de la localisation $[1,2]$ ou au mieux à valider des simulations par éléments finis [3]. À noter aussi quelques publications utilisant la tomographie par rayons $\mathrm{X}$ afin de numériser la microstructure de mousses à cellules ouvertes afin de construire des modèles éléments finis détaillés $[4,5]$. L'utilisation quantitative de mesures de champs afin d'identifier les paramètres du comportement hyperélastique de telles mousses reste donc un sujet ouvert. L'objectif de cette communication est d'apporter une contribution à ce sujet en proposant une stratégie 


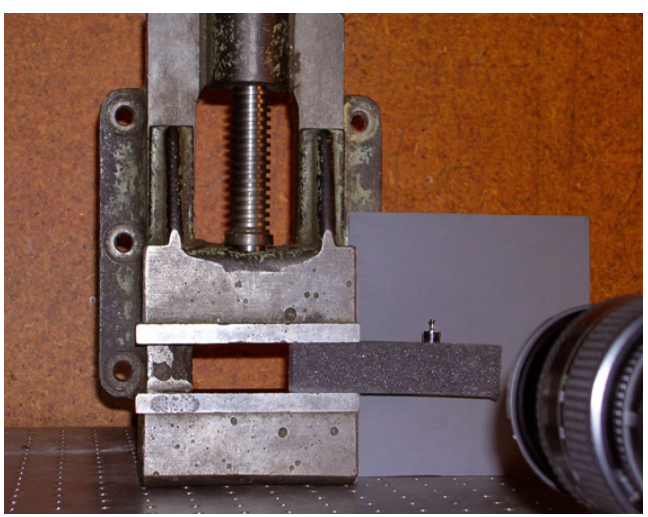

(a) vue d'ensemble

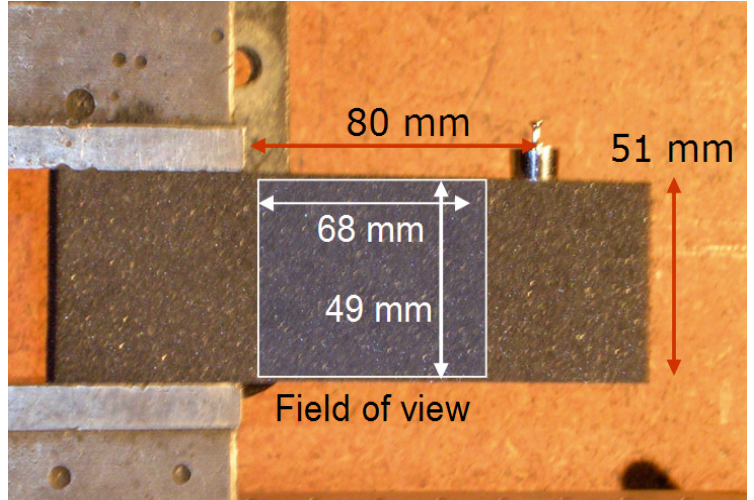

(b) détail de l'éprouvette

Fig. 1. Montage expérimental pour les paramètres élastiques.
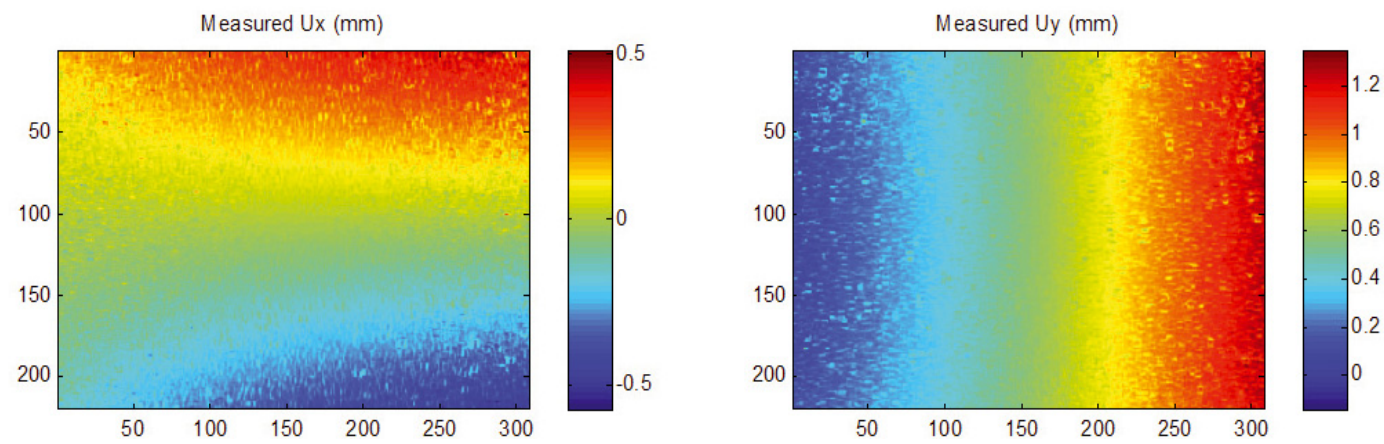

Fig. 2. Champs de déplacement pour une charge de $0,29 \mathrm{~N}$ (30 g).

fondée sur des mesures de champs de déplacement par corrélation d'images et une identification par la méthode des champs virtuels [6].

\section{Paramètres élastiques}

La première étape du travail concerne l'identification des rigidités élastiques du matériau. Celui-ci est ici une mousse polyuréthane basse densité $\left(30 \mathrm{~kg} \cdot \mathrm{m}^{-3}\right)$ de couleur grise utilisée généralement comme matériau d'emballage. En fait, cette mousse peut également servir de précurseur pour la fabrication de mousses auxétiques (coefficient de Poisson négatif, [7]). Pour cela, un montage très simple est mis en œuvre (voir Fig. 1). Un barreau rectangulaire de mousse est sollicité en flexion/cisaillement à l'aide d'une série de masses calibrées. Un encastrement sommaire est réalisé à l'aide d'un étau. Le champ de déplacement plan est mesuré à l'aide d'une caméra CCD 12-bit 1.3 Mpixels PCO Sensicam munie d'un objectif de $60 \mathrm{~mm}$ de focale. Le contraste naturel de la mousse est utilisé pour réaliser les mesures de déplacement par corrélation d'images numériques. Le logiciel Correli développé au LMT Cachan est utilisé pour cette étude [8]. Les fenêtres de corrélation utilisées sont ici de $16 \times 16$ pixels $^{2}$ avec un décalage de 8 pixels. Le résultat est un tableau de $154 \times 110$ points de mesures non indépendants (à cause du décalage de 8). La taille physique du pixel est de $55 \mu \mathrm{m}$. Les champs de déplacement sont ensuite lissés à l'aide d'une base de fonctions par morceaux de type éléments-finis (éléments bilinéaires triangulaires). Les déplacements nodaux sont déterminés par ajustement au sens des moindres carrés. Les déformations sont ensuite obtenues à l'aide des fonctions de forme puis projetées sur le même maillage (pour les détails, voir [9]).

Les champs de déplacement sont donnés en figure 2. Le bruit est important car le contraste naturel de la mousse n'est pas idéal ici. Frotter la surface à la craie blanche améliore significativement l'histogramme et supprime les points brillants. Cette procédure a été utilisée pour les essais de compression de la section suivante. La figure 3 présente les champs de déformation associés pour une taille de maille de 20 points de mesure. Plusieurs tailles de maillage ont été utilisées pour évaluer l'influence du lissage sur les paramètres identifiés. On peut voir sur cette figure qu'on est bien en présence d'un champ de flexion (voir $\varepsilon_{x x}$ )/cisaillement (voir $\varepsilon_{x y}$ ) combiné.

L'identification s'effectue à l'aide de la méthode des champs virtuels. Cette technique permet l'identification des paramètres élastiques de manière directe à partir des champs mesurés. Un point important concerne le choix des champs virtuels. Ceux-ci ont une influence importante sur la qualité de l'identification. Ici, des champs optimisés par morceaux définis sur la même base de fonction que les champs réels lissés permettent d'obtenir la solution 

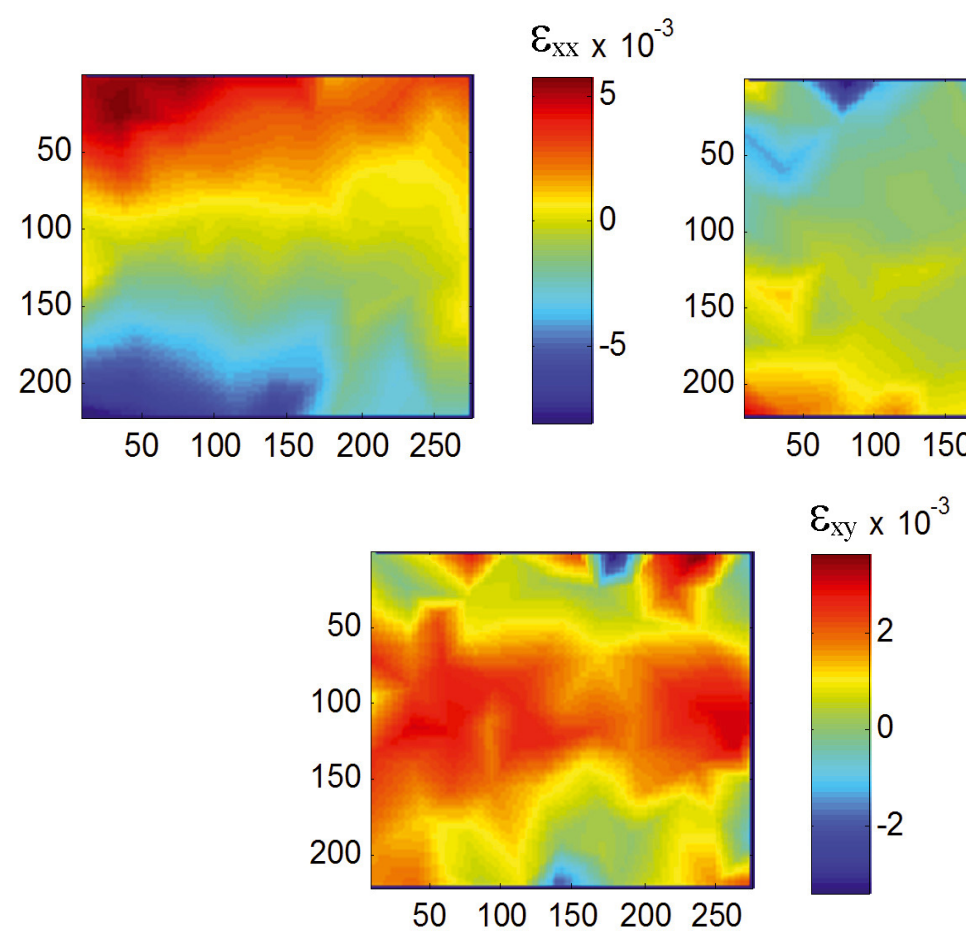

Fig. 3. Champs de déformation pour une charge de $0,29 \mathrm{~N}$ (30 g).

Tableau 1. Paramètres élastiques pour une charge de $0,29 \mathrm{~N}$ (30 g).

\begin{tabular}{lcc}
\hline Taille de maille & $E(\mathrm{kPa})$ & $\nu$ \\
\hline 12 & 398 & 0,335 \\
14 & 413 & 0,326 \\
16 & 415 & 0,346 \\
18 & 408 & 0,353 \\
20 & 417 & 0,372 \\
\hline Moyenne & 404 & 0,333 \\
\hline Coef. Var. $(\%)$ & 3,9 & 10,7 \\
\hline
\end{tabular}

au maximum de vraisemblance. Toute la procédure est détaillée dans $[9,10]$. Il faut noter que cette procédure est implémentée dans un logiciel libre téléchargeable sur www . camfit.fr. Les résultats de l'identification sont reportés dans le tableau 1. On voit que les résultats dépendent peu de la taille de maille, ce qui indique une identification stable et donc, satisfaisante. On voit que le coefficient de variation est plus élevé pour le coefficient de Poisson, ce qui est habituel car celui-ci influence moins le champ réel que le module de Young. Nous n'avons pas de valeurs de référence du module de Young pour ce matériau mais pour le coefficient de Poisson, [7] rapportent une valeur de 0,25 pour une déformation de $20 \%$. Ils montrent que ce coefficient décroît fortement avec le niveau de déformation, la valeur de 0,33 identifiée ici est donc cohérente avec leurs résultats. Il faut noter la grande simplicité de la présente procédure, la mesure de champs permettant de s'affranchir du contrôle rigoureux des conditions aux limites (de l'encastrement en particulier ici). La technique peut donc être utilisée pour obtenir rapidement des valeurs de paramètres élastiques pour ces matériaux très souples. Des essais sur mousses auxétiques sont en cours.

\section{Comportement hyperélastique}

Le vrai enjeu de la méthodologie présentée dans ce papier est de l'étendre au comportement hyperélastique en grandes transformations. Pour cela, la version nonlinéaire de la méthode des champs virtuels sera utilisée [11]. Une difficulté supplémentaire est de formuler la méthode des champs virtuels en grandes transformations. Pour cela, on utilisera une formulation dans la configuration non déformée [12]. La détermination du tenseur gradient à partir des mesures de déplacement permettra d'obtenir les élongations. On utilisera ensuite la loi d'Ogden pour déterminer les contraintes de Cauchy qu'on transformera ensuite en contraintes de Piola-Kirchhoff pour enfin appliquer le principe des travaux virtuels.

Dans un premier temps, un essai très simple de compression uniaxiale est réalisé. La figure 4 montre le montage expérimental utilisé et les dimensions de l'éprouvette.

Les mesures sont réalisées avec les mêmes paramètres que dans la section 2. Simplement, ici, la surface a été frottée à la craie (voir Sect. 2). 40 images sont enregistrées au cours de l'essai. Les élongations sont calculées après lissage par éléments-finis (cf. précédemment), avec une taille de maille de 10 points de mesures. Il faut noter ici que la corrélation se fait de manière incrémentale (i.e., l'image de référence est renouvelée à chaque pas de chargement). Ceci permet d'obtenir des niveaux de déformation très élevés (jusqu'à une compression proche 

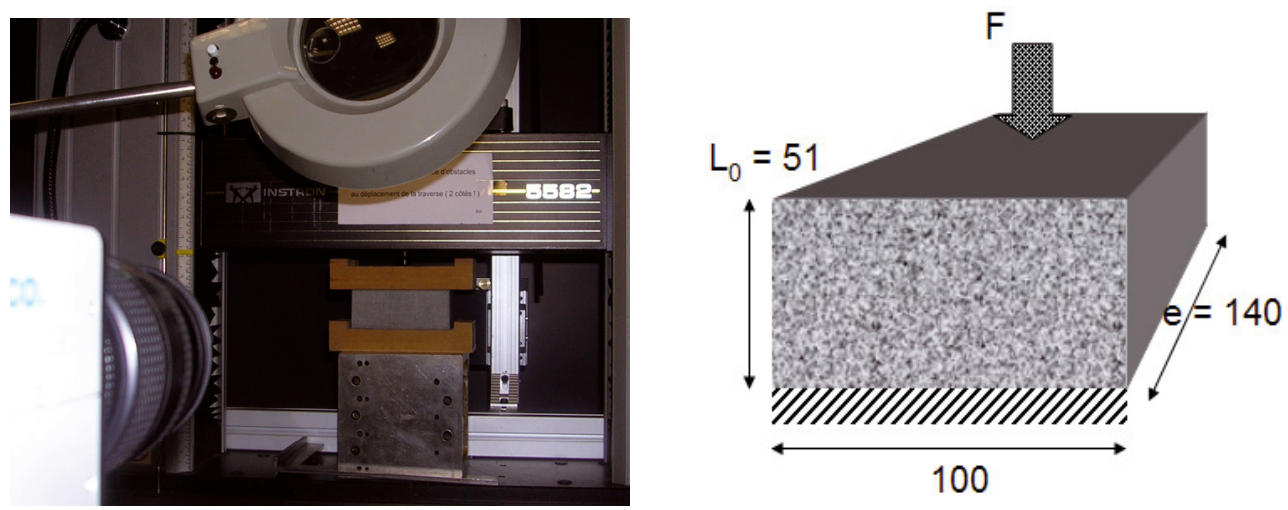

Fig. 4. Essai de compression uniaxiale (dimensions en mm).

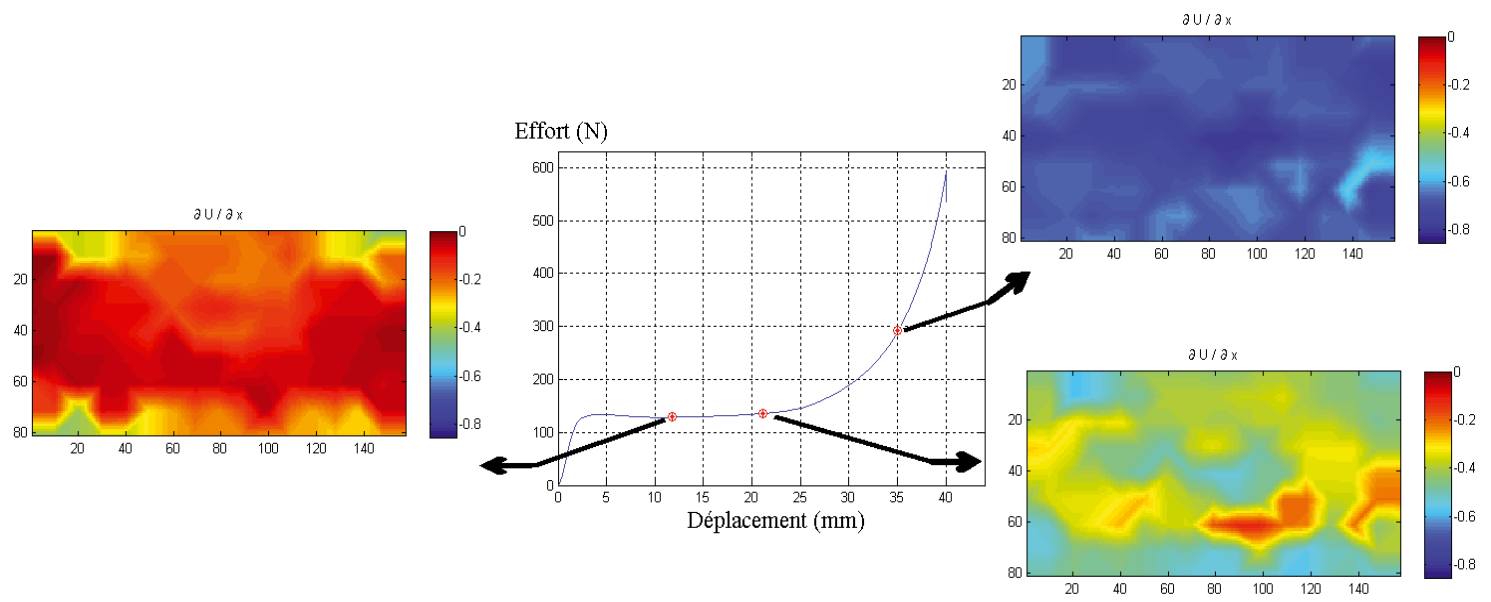

Fig. 5. Champs de déformation axiale au cours du chargement.

de 1). Les élongations de compression sont données sur la figure 5 pour différents niveaux de chargement. On voit qu'au début du plateau, une localisation s'amorce sur les faces de chargement, puis se propage au cour de l'échantillon. Après compaction, le champ redevient presque homogène.

Pour l'identification, et dans un premier souci de simplifier le problème au maximum, on suppose que le coefficient de Poisson est nul (raisonnable pour de fortes déformations, voir [7]) et que la contrainte est purement uniaxiale. Dans ces conditions, comme la section ne varie pas, la contrainte de Cauchy $\sigma_{11}$ est égale à la contrainte de Piola-Kirchhoff $\Pi_{11}$. Toujours dans un souci de simplification, on choisit une loi de comportement de type Ogden à deux paramètres :

$$
\sigma_{11}=\frac{1}{\lambda_{1}} \mu\left[\lambda_{1}^{\alpha}-1\right]
$$

où $\lambda_{1}$ est l'allongement dans la direction de compression (supposée direction principale ici) et $\mu$ et $\alpha$ les deux paramètres à identifier.

En prenant un champ virtuel de compression uniforme, on peut alors définir la fonction coût suivante :

$$
\Phi(\mu, \alpha)=\sum_{i=1}^{n}\left(F^{(i)} L_{0}-e \int \Pi_{11}^{(i)}(\mu, \alpha) \mathrm{d} x \mathrm{~d} y\right)^{2}
$$

avec $L_{0}$ la hauteur initiale de l'éprouvette et $e$ la profondeur (voir Fig. 4), $F^{(i)}$ l'effort à l'étape de chargement $i$ ( $n$ étapes en tout, ici, $n=40$ ) et $\Pi_{11}^{(i)}$ la contrainte de Piola-Kirchoff à l'étape $i$. La mesure de l'élongation $\lambda_{1}$ étant obtenue en une matrice de $p \times q$ points, on peut discrétiser l'équation précédente et substituer $\sigma_{11}$ à $\Pi_{11}$ et utiliser la loi d'Ogden :

$\Phi(\mu, \alpha)=$

$$
\sum_{i=1}^{n}\left(F^{(i)} L_{0}-\frac{e L_{0} b}{p q} \sum_{p, q} \frac{1}{\lambda_{1}^{(i)}(p, q)} \mu\left[\lambda_{1}^{(i)^{\alpha}}(p, q)-1\right]\right)^{2}
$$

Cette fonction coût est représentée en figure 6 pour une plage de valeurs assez large des paramètres de la loi d'Ogden. Un minimum est visible aux alentours de $[\mu, \alpha]=[7000 \mathrm{~Pa}, 300]$, bien qu'au-delà d'une valeur de 100 pour $\alpha$, ce paramètre n'influence presque plus la fonction coût. On voit également sur cette figure (à droite) un graphe donnant les travaux virtuels extérieurs et intérieurs (respectivement les termes de gauche et de droite dans la fonction coût) en fonction de l'élongation moyenne, ceci pour le couple de valeurs optimales $[\mu, \alpha]=$ [6800 Pa, 342]. On voit clairement que la loi choisie ne reproduit que grossièrement la réponse mais des progrès restent à faire. Il est possible d'ajouter des termes dans 

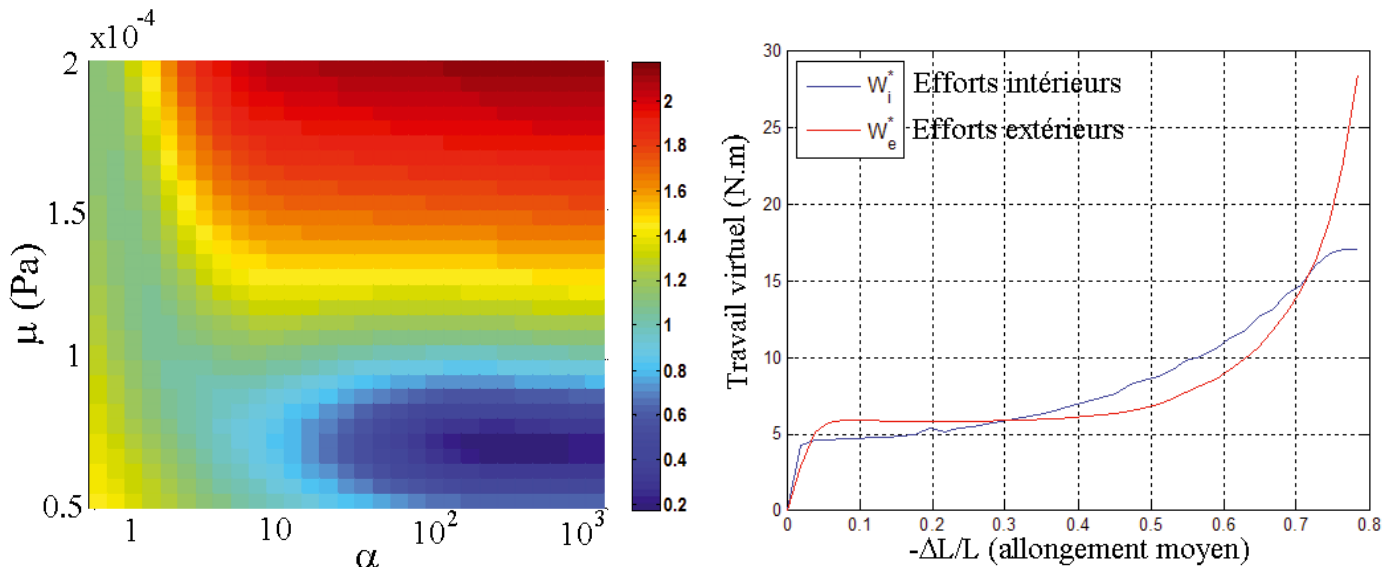

Fig. 6. Résultat de l'identification : fonction coût (gauche), qualité du modèle (droite).

cette loi pour affiner la description de la réponse, avec le risque néanmoins de se heurter à des problèmes d'unicité. Ce travail n'est qu'une première ébauche et il reste beaucoup à faire pour fiabiliser ce genre d'approche, y compris en améliorant la mesure.

\section{Conclusions}

Cette étude est une première approche pour identifier le comportement élastique et hyperélastique de mousses polymères basses densité. Les champs de déformations sont obtenus par corrélation d'images, technique parfaitement adaptée à ce type de comportement. L'implémentation incrémentale de cette technique permet ici de suivre les déformations jusqu'à presque $100 \%$, et ceci en présence de fortes variations du mouchetis au cours de l'essai. La méthode des champs virtuels permet une identification simple et rapide sans avoir à mettre en œuvre de lourdes procédures de recalage par élémentsfinis. Les résultats préliminaires sont encourageants.

Remerciements. Les auteurs remercient Fabrizio Scarpa (Université de Bristol, GB) pour la fourniture des mousses, François Hild (LMT, ENS Cachan) pour la mise à disposition du logiciel Correli ainsi que Michel Grédiac pour son aide sur la MCV en grandes transformations. Ce travail fait partie du projet ANR Blanc « Photofit » (ANR-05-BLAN-0327-01).

\section{Références}

[1] Y. Wang, A.M. Cuitiño, Full-Field measurements of heterogeneous deformation patterns on polymeric foams using digital image correlation, Int. J. Solids Struct. 39 (2002) 3777-3796

[2] R. Guastavino, P. Göransson, A 3D displacement measurement methodology for anisotropic porous cellular foam materials, Polym. Test. 26 (2007) 711-719
[3] H. Jin, W.-Y. Lu, S. Scheffel, T.D. Hinnerichs, M.K. Neilsen, Full-field characterization of mechanical behavior of polyurethane foams, Int. J. Solids Struct. 44 (2007) 6930-6944

[4] J.H. Kinney, G.W. Marshall, S.J. Marshall, D.L. Haupt, Three-dimensional imaging of large compressive deformations in elastomeric foams, J. Appl. Polym. Sci. 80 (2001) $1746-1755$

[5] J.A. Elliott, A.H. Windle, J.R. Hobdell, G. Eeckhaut, R.J. Oldman, W. Ludwig, E. Boller, P. Cloetens, J. Baruchel, In-situ deformation of an open-cell flexible polyurethane foam characterised by $3 \mathrm{D}$ computed microtomography, J. Mater. Sci. 37 (2002) 1547-1555

[6] M. Grédiac, F. Pierron, S. Avril, E. Toussaint, The virtual fields method for extracting constitutive parameters from full-field measurements: a review, Strain 42 (2006) $233-253$

[7] A. Bezazi, F. Scarpa, Mechanical behaviour of conventional and negative Poisson's ratio thermoplastic polyurethane foams under compressive cyclic loading, Int. J. Fatigue 29 (2007) 922-930

[8] F. Hild, B. Raka, M. Baudequin, S. Roux, F. Cantelaube, Multiscale displacement field measurements of compressed mineral-wool samples by digital image correlation, Appl. Opt. 41 (2002) 6815-6828

[9] S. Avril, F. Pierron, General framework for the identification of elastic constitutive parameters from full-field measurements, Int. J. Solids Struct. 44 (2007) 4978-5002

[10] S. Avril, M. Grédiac, F. Pierron, Sensitivity of the virtual fields method to noisy data, Comput. Mech. 34 (2004) 439-452

[11] S. Avril, F. Pierron, Y. Pannier, R. Rotinat, Stress reconstruction and constitutive parameter identification in elastoplasticity using measurements of deformation fields, Exp. Mech. 2008. Sous presse

[12] F. Sidoroff, Cours sur les grandes déformations, rapport GRECO n $51 / 1982$ 